\title{
Identification of a crack propagation law by digital image correlation
}

\author{
Florent Mathieu ${ }^{\mathrm{a}}$, François Hild ${ }^{\mathrm{a}}$, Stéphane Roux ${ }^{\mathrm{a}}$ \\ ${ }^{a}$ Laboratoire de Mécanique et Technologie (LMT-Cachan) \\ ENS de Cachan / UMR CNRS / Université Paris 6 / PRES UniverSud Paris \\ 61 Avenue du Président Wilson, F-94235 Cachan Cedex, France
}

\begin{abstract}
It is proposed to use digital image correlation (DIC) to identify parameters governing crack propagation of commercially pure titanium. To achieve this goal, crack tip location, stress intensity factor, $T$-stress and plastic zone size are sought. Most of the DIC approaches are based upon local analyses of displacements, and their subsequent projection onto a set of mechanically relevant fields. It is proposed to perform these two sequential steps in a unique (and integrated) way, and to compare the results with a global approach to DIC with subsequent post-processing. A priori performances of two global approaches are compared, and a propagation law is identified form the series of raw images of a fatigue test on commercially pure titanium with the integrated approach that yields better results.
\end{abstract}

\section{Introduction}

Since the first attempt to measure a stress intensity factor (SIF) by digital image correlation [1], a lot of improvements have appeared. DIC is definitely a tool of choice to analyze cracked samples and structures [2]. In particular,

Email addresses: florent.mathieu@lmt.ens-cachan.fr (Florent Mathieu), francois.hild@lmt.ens-cachan.fr (François Hild), stephane.roux@lmt.ens-cachan.fr (Stéphane Roux) 
for cyclic tests, one key aspect is related to the estimation of crack opening and closure levels when studying the propagation conditions. The latter ones could be determined by analyzing crack opening displacements measured by DIC $[3,4]$. These conditions could also be studied thanks to the analysis of SIF histories [5]. Crack propagation laws were also determined when the crack tip position was optically determined when using a long-distance microscope [7], or by using the crack opening displacements [8]. In the following, another will be followed to determine the crack tip position. When the propagation path is not straight, edge detection procedures can also be used to determine the shape of the crack [9], or even the correlation residuals themselves when a global approach to DIC is used [10].

Some of the previous aspects can also be studied thanks to computed tomography and in situ tests when resorting to digital volume correlation [11]. In particular, stress intensity factors along the crack front and crack opening displacement maps allowed the same authors to analyze the closure conditions in a fatigue experiment on nodular graphite cast iron [12], and its influence of the propagation conditions [13]. Even though most of the tools developed herein could be extended to deal with 3D situations, the analysis detailed herein is applied to 2D pictures and aims at the direct and automated determination of crack propagation laws based upon the sole use of digital images.

When analyzing initiation conditions, DIC was also used on meso [14] and microscales $[15,16]$ for which multiple damage sites occur. Damage mechanisms could be analyzed in a quantitative manner when using the measured displacement fields. When combined with infrared thermography, heat sources and stress levels are identified thanks to inverse thermomechanical analyses [17]. Ad hoc correlation procedure was also developed to count automatically the number of initiated mesocracks [18]. This information is then used to tune the parameters of a continuous initiation model [19]. In the following, only one long crack 
will be analyzed.

One key parameter driving crack propagation is the SIF. Most of the approaches to measure an SIF are based on post-processing the measured displacement fields to extract stress intensity factors [20,21, 22]. Least squares minimization is generally used when the measured data are projected onto reference (i.e. mechanically admissible [23]) fields. The amplitudes associated with these reference fields give access, for instance, to SIFs. Alternative extracting techniques follow the same path as for numerical simulations (e.g. the interaction integral [24]). The latter can be optimized to be the least sensitive to measurement uncertainties [25]. Recently, it was shown that the interaction integral is only a local minimizer, and that optimal SIF extractors deal with the measured data, namely, the displacement field, and not any of their derivatives since they will be less sensitive to measurement uncertainties [26].

The first question to address when evaluating an SIF is related to the location of the crack tip. This is clearly an experimental challenge since the experimentalist cannot rely on a manual estimate that neglects sub-pixel crack openings. Most of the time, the location of the crack tip is part of the minimization procedure, which becomes nonlinear. Another route was proposed by Hamam et al. [5]. It consists in considering so-called supersingular displacement fields, and more precisely, the first one that is usually discarded in a purely elastic analysis. The latter one can be used to locate the crack tip by canceling out its amplitude. This type of approach was applied both on numerical simulations [27], and experimental results $[5,28,2,26]$. It will be used herein to follow the crack tip in a cyclic test during which propagation occurs.

To evaluate directly (i.e. with no post-processing of the measured displacement field) the SIF and thus the crack tip location and other relevant mechanical parameters, so-called "integrated approaches" of DIC (or I-DIC [29, 30, 5, 28]) can be followed. In a global approach to DIC, it consists in implementing di- 
rectly the relevant fields when minimizing the functional describing the gray level conservation. This approach will be used herein to evaluate SIFs, crack tip locations, $T$-stress components (i.e. the stress level parallel to the crack faces) on a thin cracked sheet made of commercially pure titanium. One of the outputs will be the parameters of a Paris' law [31]. Plastic zone size and shape will also be discussed.

In the following section, the experimental configuration is described, and followed by the presentation of two SIF extraction techniques. An a priori analysis allows for the evaluation of the performances of both techniques. Last, experimental results on commercially-pure titanium are discussed.

\section{Experimental configuration}

The aim of the present study is to determine the parameters of a crack propagation law for thin sheets of so-called Ti35 (i.e. commercially-pure titanium, max 0.2wt\% Fe, 0.18wt\% O, 0.08wt\% C, 0.03wt\% N, 0.015wt\% H). A $0.3 \mathrm{~mm}$ thick center-cracked sample is subjected to a cyclic tensile load inducing the propagation of two crack tips. Pictures are shot regularly for the analysis proposed herein. The sample is observed by a single camera (definition: $1022 \times 1024$ pixels, digitization: 12 bits) and a telecentric lens focused on the vicinity of one of the notch tips. The lens allows us to minimize (if not completely cancel out) artifacts related to out-of-plane motions.

To observe crack propagation, a cyclic fatigue test is performed along the longitudinal direction of forming (load ratio: $R=0.1$, load frequency: $f=$ $10 \mathrm{~Hz}$ ). As shown in Figure 1(a), the images are taken every 1000 cycles up to failure of the sample, for the minimum and maximum load levels. The maximum force $F$ corresponds to approximately $120 \mathrm{MPa}$ of remote stress (i.e. about $50 \%$ of the initial yield stress $\sigma_{y}$ of the tested material). The center-cracked sample is shown in Figure 1(b). The propagation is studied only on one side of the 
crack. Sample failure occurs around 120000 cycles, while crack propagation is observable during the last 60000 cycles.

\section{Parameter extraction}

The sample being observed with a telecentric lens (Figure 2) a physical size of $6.1 \mu \mathrm{m}$ for each pixel is achieved. The image in the deformed configuration $g$ is related to that in the reference configuration $f$ by assuming gray level conservation

$$
f(\mathbf{x})=g(\mathbf{x}+\mathbf{u}(\mathbf{x}))
$$

where $\mathbf{u}$ is the sought displacement field. As such, the measurement problem is ill-posed. A global approach consists in minimizing the (global) residual between the reference image $f$ and the deformed image corrected by the displacement field $\tilde{g}(\mathbf{x})=g(\mathbf{x}+\mathbf{u}(\mathbf{x}))$ over the whole region of interest $\Omega$

$$
\eta^{2}=\iint_{\Omega}[g(\mathbf{x}+\mathbf{u}(\mathbf{x}))-f(\mathbf{x})]^{2} \mathrm{~d} \mathbf{x}
$$

The form of the displacement field $\mathbf{u}$ can be chosen by the user. For instance, it may be decomposed over a basis of shape functions $N_{m}$ of general form

$$
\mathbf{u}(\mathbf{x})=\sum_{\alpha, m} v_{\alpha m} N_{m}(\mathbf{x}) \mathbf{e}_{\alpha}
$$

where $v_{\alpha m}$ are the unknown degrees of freedom, and $\mathbf{e}_{\alpha}$ is a unit vector of the element frame. In the following, 4-noded elements are considered (i.e. a Q4-DIC approach [32]).

Conversely, a series of mechanically relevant fields $\psi_{n}$ (e.g. Williams' series [23])

$$
\mathbf{u}(\mathbf{x})=\sum_{n} w_{n} \boldsymbol{\psi}_{n}(\mathbf{x})
$$


can be chosen, where $w_{n}$ are generalized degrees of freedom (e.g. mode I and II SIFs).

\subsection{Post-processing approach}

The first DIC procedure consists in measuring displacement fields discretized with Q4 elements. The element size was chosen to be equal to 16 pixels. This value is a good compromise between measurement uncertainty and spatial resolution [32]. No enrichment was used [10, 28] in the present case since the crack tip and path will be masked. A measurement result is shown in Figure 3. The displacement field is subsequently post-processed (via least squares fit) by using reference fields, namely, Williams' series relevant for small scale yielding when the latter region is excluded from the analysis, by resorting to a least squares technique $[1,21,22,5]$. These fields $\mathbf{u}=u_{x}+i u_{y}$ take the following expression in the crack frame (crack tip at the origin, and crack path along the negative $x$ axis) resorting to the complex plane, $z=r \exp (i \theta)$

$$
\mathbf{u}(z)=\sum_{n}\left[\omega_{n} \boldsymbol{\Omega}_{n}(z)+v_{n} \boldsymbol{\Upsilon}_{n}(z)\right]
$$

with, for a mode I regime

$$
\begin{aligned}
\boldsymbol{\Omega}_{n}(z) & =\frac{(-1)^{(1-n) / 2}}{2 \mu \sqrt{2 \pi}} r^{n / 2}\left[\kappa \exp \left(\frac{i n \theta}{2}\right)-\frac{n}{2} \exp \left(\frac{i(4-n) \theta}{2}\right)\right. \\
& \left.+\left((-1)^{n}+\frac{n}{2}\right) \exp \left(-\frac{i n \theta}{2}\right)\right]
\end{aligned}
$$

and a mode II regime

$$
\begin{aligned}
\Upsilon_{n}(z) & =\frac{i(-1)^{(1-n) / 2}}{2 \mu \sqrt{2 \pi}} r^{n / 2}\left[\kappa \exp \left(\frac{i n \theta}{2}\right)+\frac{n}{2} \exp \left(\frac{i(4-n) \theta}{2}\right)\right. \\
& \left.+\left((-1)^{n}-\frac{n}{2}\right) \exp \left(-\frac{i n \theta}{2}\right)\right]
\end{aligned}
$$

where $\mu$ is Lamé's modulus, and $\kappa$ a dimensionless parameter dependent on Poisson's ratio $\nu$ (i.e. $\kappa=(3-\nu) /(1+\nu)$ in plane stress, or $3-4 \nu$ in plane 
strain). Due to the very small thickness of the considered sample, a plane stress solution is assumed to be a good approximation of the in-plane fields.

Amplitudes $\omega_{1}$ and $v_{1}$, associated with fields $\boldsymbol{\Omega}_{1}$ and $\boldsymbol{\Upsilon}_{1}$, are the mode I and II SIFs, $K_{I}$ and $K_{I I}$, respectively. Amplitudes $\omega_{0}$ and $v_{0}$ correspond to rigid body translations. Amplitudes $\omega_{2}$ and $v_{2}$ give access to the $T$-stress component, and the rigid body rotation. The crack tip is located by canceling out amplitude $\omega_{-1}$ of the first supersingular field. More precisely, a non-zero $\omega_{-1}$ results in a shift $d$ of the crack tip with respect to its exact location [2]

$$
d=\frac{2 \omega_{-1}}{\omega_{1}}
$$

The use of supersingular fields allows us to account for local nonlinear effects. For this purpose, $n_{\min }=-3$ seems relevant, since lower order fields show very small displacements in the analyzed zone (see Section 5.6). On the other hand, subsingular fields account for large-scale effects, such as boundary conditions. In practice, $n_{\max }$ ranging from 5 to 8 is sufficient. In this analysis $n_{\max }=5$ was chosen to limit the number of fields used, which allows for faster computations.

The extracted fields are compared with the raw measurement as shown in Figure 6. A good agreement is observed. The root mean square (RMS) residual between the two fields is equal to 0.05 pixel. This value is (only) 2 times that of the resolution of the measurement technique for the selected element size, which is of the order of 0.025 pixel. To obtain this value, an artificial image is built by artificially applying to the reference image a 0.5 -pixel motion in both directions. The uncertainty is the standard deviation of the measured field [32].

The post-processing approach is very fast, but it relies on measured displacement fields that are not expressed in the final kinematic basis. This intermediate step introduces an additional uncertainty to the computation of SIFs. This is why integrated approaches are proposed to perform in a single step the measurement and identification procedures $[30,5]$. 


\subsection{Integrated approach}

In this approach, the displacement field is directly decomposed over a kinematic basis whose components are those of the mechanically relevant fields [see Equation (5)]. The sought mechanical parameters (i.e. $\omega_{n}$ and $v_{n}$ ) are the generalized degrees of freedom of the chosen displacement fields that are measured from the raw images (Figure 6). This approach avoids one step of the former method that may introduce an additional uncertainty to the evaluation of $\omega_{n}$ and $v_{n}$. The SIFs and the crack tip location are therefore direct outputs of the integrated approach.

The crack tip location is one of the unknowns of the global correlation residual minimization [Equation (2)]. Consequently, I-DIC requires a longer computation time since the global matrix to be inverted has to be recalculated each time the crack tip is moved to a new position. The computation time takes about 1 hour for the whole post-processing approach (i.e. a series of 60 images, with a Matlab ${ }^{\circledR}$ code), and the integrated approach is performed in about 3 to 5 hours, depending on the value of the convergence criterion in terms of crack tip increment.

\section{Baseline analysis}

To evaluate the performances of both techniques, a test case was run in which a reference image was artificially deformed using a pure mode I field with various amplitudes. The displacement amplitudes (i.e. from $3 \times 10^{-3}$ to 16 pixels) would correspond approximately to 0.01 to $55 \mathrm{MPa} \sqrt{\mathrm{m}}$ for the tested material in the present configuration. Both extraction techniques are applied to these artificially deformed images with the same mask parameters (inner radius and frame of 10 pixels, external radius of 400 pixels).

Results of $K_{I}$ measurements are shown in Figure 4(a). While SIF measurements are generally in good agreement with prescribed values, I-DIC shows a 
particularly good performance in terms of SIF values. The post-processing route leads to a poorer resolution (i.e. the "smallest change in a quantity being measured that causes a perceptible change in the corresponding indication' [33]). The error with respect to the prescribed SIF is shown in Figure 4(b). For a prescribed SIF greater than $0.05 \mathrm{MPa} \sqrt{\mathrm{m}}$, the error is less than $8 \times 10^{-4} \mathrm{MPa} \sqrt{\mathrm{m}}$ for I-DIC. For the post-processing route, values can be trusted for SIFs greater than $1 \mathrm{MPa} \sqrt{\mathrm{m}}$ so that the error is less than $0.1 \mathrm{MPa} \sqrt{\mathrm{m}}$. This difference can be justified by the fact that much fewer degrees of freedom are used in the I-DIC approach.

Figure 5 displays the distance between the prescribed and identified crack tip position in pixels. The convergence criterion, which is written in terms of offset $d$ defined in Equation (8), has been set to 0.5 pixel. Both techniques show good agreement for measured SIFs greater than $0.1 \mathrm{MPa} \sqrt{\mathrm{m}}$ for the studied material parameters. I-DIC provides a quasi constant crack tip position for these values of SIF (mean offset of 0.74 pixel with a standard deviation of 0.17 pixel, which is below the convergence criterion), while the post-processing route shows more scatter (mean offset of 5.5 pixels with a standard deviation of more than 3.5 pixels).

From this analysis, it can be concluded that I-DIC has a better performance than that provided by the post-processing route. For larger levels of SIF, both techniques yield similar results. It is worth noting that special care should be exercised when SIF evaluation is addressed. The value of the latter is directly related to the correct positioning of the crack tip. A slight offset has a direct effect on the SIF estimate. 


\section{Experimental results}

\subsection{Displacement and correlation residual fields}

Figure 6 shows the displacement fields between minimum and maximum loading after 60000 cycles. For an assumed crack tip location, the correlation residual $\eta^{2}$ is minimized within the region of interest, which is the zone over which the fields are plotted. The external radius of this mask turns out to have little influence on the measurement results provided it is large enough to encompass a significant zone; the internal radius and the box around the crack must be kept to a minimum value (i.e. 50 pixels in the present case) in order not to consider regions affected by plastic strains in the vicinity of the crack tip. The correlation residuals are shown in Figure 7(a). Their level remains very low, i.e. of the order of 2 percent of the dynamic range of the reference picture in the considered zone for I-DIC, and less than 4 percent at most for postprocessed Q4-DIC (Figure 7(b)). These low values indicate that the registration was successful, and that the measured displacements are trustworthy. It can be noted that in the immediate vicinity of the crack front the registration would have been less successful, had the corresponding region not been masked. The fact that the mean level is virtually independent of the number of cycles for I-DIC (Figure 7(b)) shows that except for the very last points, the proposed framework is validated.

\subsection{Crack tip position and stress intensity factor}

Having determined amplitudes $\omega_{n}$ and $v_{n}$ for all the considered pictures, it is possible to plot the change of the SIF amplitude $\Delta K$ with the crack length increment $\Delta a$ (Figure $8(\mathrm{~b}))$, and the latter one with the number of cycles (Figure $8(\mathrm{a}))$. The SIF estimate is compared to the tabulated values for a CCT specimen [34] by considering the same crack tip position as that determined by the integrated approach and a symmetric propagation 


$$
K=\frac{F \sqrt{\pi a}}{2 b W}\left[1-0.025\left(\frac{a}{W}\right)^{2}+0.06\left(\frac{a}{W}\right)^{4}\right] \sqrt{\frac{1}{\cos \left(\frac{\pi a}{2 W}\right)}}
$$

where $F$ is the applied load, $b$ the thickness of the sample, $W$ its width, and $a$ the length of the center crack. A good agreement is observed except at the end of the experiment for which large scale yielding is likely to occur, or buckling of the sample under the resulting transverse compression. The SIF offset at the beginning of propagation may be explained by the fact that only one crack tip was observed, and therefore a symmetry hypothesis had to be made.

\subsection{Paris' law determination}

The previous results allow us to determine the parameters of the crack propagation law when described by a Paris' model. The latter is expressed with or without SIF threshold as

$$
\begin{gathered}
\frac{d a}{d N}=A \Delta K^{n} \\
\frac{d a}{d N}=B\left(\Delta K-\Delta K_{t h}\right)^{p}
\end{gathered}
$$

where $\Delta K_{t h}$ denotes the threshold SIF amplitude below which no propagation occurs.

Instead of tuning the parameters directly between the measured SIF range and the numerical crack velocity $d a / d N$, which can be very noisy, the growth law is recast in terms of the crack growth increment $\Delta a$

$$
\Delta a(N)=\int_{0}^{N} B\left(\Delta K-\Delta K_{t h}\right)^{p} d N
$$

This quantity is numerically evaluated when $\Delta K$ itself is assumed to vary with $\Delta a$ as

$$
\Delta K=C e^{D \Delta a+E \Delta a^{2}}
$$

where $C, D$ and $E$ are parameters to be tuned. This form is chosen because it provides a good compromise between a small sensitivity to measurement uncertainties and has a small number of parameters (i.e. 3), see Figure 9. By 
comparing the numerically evaluated $\Delta a$ [Equation (12)] to the measured values, it is possible to determine the best parameters $B, K_{t h}$ and $p$ of Paris' law. Direct fit between the measured SIF range and the crack growth rate $d a / d N$ did not yield such good agreement between the measured and the identified crack growth increment. Figure 9 shows the results obtained for the test. Values of the identified parameters are given in Table 1. Even though the fit quality is better for I-DIC results, very similar results in terms of the propagation parameters are obtained. This is presumably fortuitous.

Figure 10 shows plots of Paris' law with and without threshold. The results obtained herein for Paris' law with no threshold [Equation (10)] are in close agreement with those reported on a different titanium grade [35]. It is not possible to distinguish from the experimental data reported herein the two types of Paris' law. Both give a good fit quality. Consequently, noting that Equation (10) is a particular case of Equation (11), i.e. $p=n, B=A, \Delta K_{t h}=0$, it means a higher uncertainty on the threshold $\Delta K_{t h}$ as the latter's effect can be compensated by a change of the other parameters.

\subsection{T-stress measurement}

The $T$-stress component (i.e. the nonsingular term in Williams' series for cracks) is another contribution of Williams' series that can be measured thanks to Q4-DIC or I-DIC, since it is directly related to $\omega_{2}$. Its estimate is compared to that proposed by McClintock [36] for a CCT specimen by considering the same crack tip position as that determined by the integrated approach and a symmetric propagation

$$
\frac{T}{\sigma_{\infty}}=-0.007+0.283 \frac{a}{W}-3.268\left(\frac{a}{W}\right)^{2}+6.622\left(\frac{a}{W}\right)^{3}-5.995\left(\frac{a}{W}\right)^{4}
$$

with

$$
\sigma_{\infty}=\frac{F}{b W}
$$


where $\sigma_{\infty}$ is the remote stress. The result presented in Figure 11 shows that there is a very good agreement with the analytical prediction. The propagation during the experiment occurs essentially with a constant $T$-stress of the order of $-120 \mathrm{MPa}$. However, at the end of the experiment, large scale yielding induces a change of the $T$-stress level, or a lack of reliability of the present analysis if buckling takes place.

\subsection{Plastic zone size}

The computation of the stress field corresponding to the measured displacement field [37] allows us to estimate the plastic zone shape, as the zone where the equivalent Von Mises' stress is greater than twice the yield stress. This estimation does not account for stress redistributions around the plastic zone. The contributions of $K_{I}, T$-stress and all the fields are analyzed in Figure 12(a). A significant difference is observed. The shape of the plastic zone is mainly due to the $n=-3$ field. When all the fields are considered except $n=-3$, the estimated plastic zone is nearly the same as with only the $K_{I}$ and $T$-stress contributions.

Within the framework of small scale yielding, an estimate of the process zone size depends upon the yield stress of the studied material. In the case of cyclic loading, this estimation reads [38]

$$
r_{p}=\frac{1}{\pi}\left(\frac{(1-R) K_{I}}{2 \sigma_{y}}\right)^{2}
$$

where $r_{p}$ is the radius of the plastic zone. Furthermore, the identified fields also give an indication of the process zone size $[2,27,26]$. The first mode I supersingular field (i.e. $n=-1$ ) gives an indication of the crack tip position along its propagation direction, the second (i.e. $n=-3$ ) provides a contribution that can be interpreted in terms of the process zone size, via a term $R_{p}$ homogeneous 
to a length scale

$$
R_{p} \propto \sqrt{-8 \frac{\omega_{-3}}{\omega_{1}}}
$$

where $\omega_{i}$ are amplitudes of order $i$ of the mode I fields (6). These different estimates are plotted all along the propagation in Figure 12(b). This dimensional indication needs to be adjusted to a known set of data. Over the first 40 images, the slope is very similar, and one can extract a proportionality coefficient of 5.1 between these two estimates. This adjustment is plotted in Figure 12(b). Let us finally note that the post-processing route did not provide trustworthy results.

\subsection{Contribution of different fields}

In order to evaluate which of the measured orders dominate over the other ones, the absolute value of the longitudinal displacement fields (6) and (7) are analyzed in the ligament in front of the crack (i.e. for $\theta=0$ ). This plot is presented in Figure 13. The gray background indicates the masked zone, since the analysis region consist of an annulus $50<r<400$ pixels.

Orders ranging from $n=-3$ to $n=5$ are shown. Since this problem is mostly in a mode I regime (it was checked that the mode II contributions are at least one order of magnitude less than those in mode I), only the mode I fields are shown. Except for order $n=1$, one can clearly distinguish subsingular fields, which have a positive slope in a log-log plot, from supersingular fields with a negative slope. The field of order $n=0$ is not shown since it corresponds to rigid body translation, which has no effect on crack propagation.

The displacement amplitudes $\omega_{n} r^{n / 2}$ (expressed in pixels) are dominant first when $n=1$, which corresponds to the usual $K_{I}$ field, and for $n=2$, which is representative of the $T$-stress contribution. This result shows that the DIC analyses were preformed in a $K_{I}$-dominant zone. Furthermore, it shows that the estimates of SIF and $T$-stress are extracted from the two dominant fields. Conversely, one can note that the amplitude of order $n=-3$, which is used 
to evaluate the process zone size [see Equation (17)], is only the third most influential field. This may explain why its estimate becomes less accurate. A higher magnification is desirable if this field is sought. The present distribution of amplitudes, only shown for a given picture, is very stable and is observed for all the 60 images analyzed herein.

\section{Conclusions}

An integrated digital image correlation (I-DIC) procedure, which allows for the direct identification of various fracture mechanics parameters, was used to tune parameters of a crack propagation law directly from the analysis of a series of 60 pairs of images of a crack propagation test on commercially-pure titanium. I-DIC was first validated against a more classical post-processing route. The former out-performed the latter in terms of correlation residuals, and resolution for stress intensity factor measurement and crack tip positioning.

This work shows that the identification of a crack propagation law is possible from one single test, provided the crack is observed during all its propagation. It requires to estimate the crack tip position. This was achieved by resorting to the first supersingular term of Williams' series whose amplitude cancels out for the most appropriate crack tip location. Once the crack tip was determined, the crack growth increment is estimated in addition to other global parameters such as the stress intensity factor range, and $T$-stress. By analyzing the dominant displacement fields, it can also be concluded that the estimate of the plastic zone size is more difficult and would require analyses with a higher magnification, or even combined kinematic and thermal measurements [39].

Last, for the end of the propagation analyzed herein, because of the submillimeter thickness of the sample the out-of-plane effects as well as local buckling may induce deviations from a purely in-plane analysis as performed herein. To check these effects, 3D-DIC [40] is a solution of choice to consider. 


\section{Acknowledgements}

This work was supported by Region Ile de France (FRESCORT project), and by Agence Nationale de la Recherche through the RUPXCUBE grant (ANR09-BLAN-0009-01). 


\section{References}

[1] S. R. McNeill, W. H. Peters and M. A. Sutton, Estimation of stress intensity factor by digital image correlation, Eng. Fract. Mech. 28 [1] (1987) 101-112.

[2] S. Roux, J. Réthoré and F. Hild, Digital Image Correlation and Fracture: An Advanced Technique for Estimating Stress Intensity Factors of 2D and 3D Cracks, J. Phys. D: Appl. Phys. 42 (2009) 214004.

[3] W. T. Riddel, R. S. Piascik, M. A. Sutton, W. Zhao, S. R. McNeill and J. D. Helm, Determining fatigue crack opening loads from near-crack-tip displacement measurements, in: Advances in fatigue crack closure measurement and analysis: second volume, STP 1343, R. C. McClung and J. Newman, J.C., eds., (ASTM, 1999 157-174).

[4] J. Carroll, C. Efstathiou, J. Lambros, H. Sehitoglu, R. Hauber, S. Spottswood and R. Chona, Investigation of Fatigue Crack Closure using Mulitscale Image Correlation Experiments, Eng. Fract. Mech. 76 (2009) 2384-2398.

[5] R. Hamam, F. Hild and S. Roux, Stress intensity factor gauging by digital image correlation: Application in cyclic fatigue, Strain 43 (2007) 181-192.

[6] P. F. P. de Matos and D. Nowell, Experimental and numerical investigation of thickness effects in plasticity-induced fatigue crack closure, Int. J. Fat. 31 (2009) 1795-1804.

[7] P. F. P. de Matos and D. Nowell, Experimental and numerical investigation of thickness effects in plasticity-induced fatigue crack closure, Int. J. Fat. 31 (2009) 1795-1804.

[8] E. Durif, M. Fregonese, J. Réthoré and A. Combescure, Development of a Digital Image Correlation controlled fatigue crack propagation experiment, 
in: F. Brémand, ed., Proceedings ICEM 14, (EPJ Web of Conferences, 2010), 7 p.

[9] S. Vanlanduit, J. Vanherzeele, R. Longo and P. Guillaume, A digital image correlation method for fatigue test experiments, Optics Lasers Eng. 47 (2009) 371-378.

[10] J. Réthoré, F. Hild and S. Roux, Extended digital image correlation with crack shape optimization, Int. J. Num. Meth. Eng. 73 [2] (2008) 248-272

[11] N. Limodin, J. Réthoré, J.-Y. Buffière, F. Hild, W. Ludwig, J. Rannou and S. Roux, 3D X-ray microtomography volume correlation to study fatigue crack growth, Adv. Eng. Mat. 13 [3] (2011) 186-193.

[12] N. Limodin, J. Réthoré, J.-Y. Buffière, A. Gravouil, F. Hild and S. Roux, Crack closure and stress intensity factor measurements in nodular graphite cast iron using 3D correlation of laboratory $\mathrm{X}$ ray microtomography images, Acta Mat. 57 [14] (2009) 4090-4101.

[13] N. Limodin, J. Réthoré, J.-Y. Buffière, F. Hild, S. Roux, W. Ludwig, J. Rannou and A. Gravouil, Influence of closure on the 3D propagation of fatigue cracks in a nodular cast iron investigated by X-ray tomography and 3D Volume Correlation, Acta Mat. 58 [8] (2010) 2957-2967.

[14] M. Poncelet, G. Barbier, B. Raka, S. Courtin, R. Desmorat, J. C. Le-Roux and L. Vincent, Biaxial High Cycle Fatigue of a type 304L stainless steel: Cyclic strains and crack initiation detection by digital image correlation, Eur. J. Mech. A/Solids 29 (2010) 810-825.

[15] A. El Bartali, V. Aubin and S. Degallaix, Fatigue damage analysis in a duplex stainless steel by digital image correlation technique, Fat. Fract. Eng. Mat. Struct. 31 [2] (2008) 137-151. 
[16] T. Niendorf, J. Dadda, D. Canadinc, H. J. Maier and I. Karaman, Monitoring the fatigue-induced damage evolution in ultrafine-grained interstitialfree steel utilizing digital image correlation, Mat. Sci. Eng. A 517 (2009) 225-234.

[17] A. Chrysochoos, B. Berthel, F. Latourte, A. Galtier, S. Pagano and B. Wattrisse, Local energy analysis of high-cycle fatigue using digital image correlation and infrared thermography, J. Strain Analysis 43 [6] (2008) 411-422.

[18] J. Rupil, S. Roux, F. Hild and L. Vincent, Fatigue microcrack detection with digital image correlation, J. Strain Analysis [accepted] (2011).

[19] J. Rupil, F. Hild, L. Vincent and S. Roux, Probabilistic modeling of mesocrack initiations in 304L stainless steel, Int. J. Multiscale Comput. Eng. [accepted] (2011).

[20] M. A. Sutton, S. R. McNeill, J. D. Helm and Y. J. Chao, Advances in Two-Dimensional and Three-Dimensional Computer Vision, in: Photomechanics, P. K. Rastogi, eds., (Springer, Berlin (Germany), 2000), Topics in Appl. Phys., 77 323-372.

[21] J. Abanto-Bueno and J. Lambros, Investigation of crack growth in functionally graded materials using digital image correlation, Eng. Fract. Mech. 69 (2002) 1695-1711.

[22] S. Yoneyama, T. Ogawa and Y. Kobayashi, Evaluating mixed-modestress intensity factors from full-field displacement fields obtained by optical methods, Eng. Fract. Mech. 74 (2007) 1399-1412.

[23] M. L. Williams, On the stress distribution at the base of a stationary crack, ASME J. Appl. Mech. 24 (1957) 109-114. 
[24] J. Réthoré, A. Gravouil, F. Morestin and A. Combescure, Estimation of mixed-mode stress intensity factors using digital image correlation and an interaction integral, Int. J. Fract. 132 (2005) 65-79.

[25] J. Réthoré, S. Roux and F. Hild, Noise-robust Stress Intensity Factor Determination from Kinematic Field Measurements, Eng. Fract. Mech. 75 [13] (2008) 3763-3781.

[26] J. Réthoré, S. Roux and F. Hild, Optimal and noise-robust extraction of Fracture Mechanics parameters from kinematic measurements, Eng. Fract. Mech. 78 [9] (2011) 1827-1845.

[27] C. Henninger, S. Roux and F. Hild, Enriched kinematic fields of cracked structures, Int. J. Solids Struct. 47 (2010) 3305-3316.

[28] J. Réthoré, S. Roux and F. Hild, An extended and integrated digital image correlation technique applied to the analysis fractured samples, Eur. J. Comput. Mech. 18 (2009) 285-306.

[29] F. Hild and S. Roux, Digital image correlation: From measurement to identification of elastic properties - A review, Strain 42 (2006) 69-80.

[30] S. Roux and F. Hild, Stress intensity factor measurements from digital image correlation: Post-processing and integrated approaches, Int. J. Fract. 140 [1-4] (2006) 141-157.

[31] P. C. Paris, M. P. Gomez and W. P. Anderson, A Critical Analysis of Crack Propagation Laws, The Trend in Engineering 13 (1961) 9-14.

[32] G. Besnard, F. Hild and S. Roux, "Finite-element" displacement fields analysis from digital images: Application to Portevin-Le Châtelier bands, Exp. Mech. 46 (2006) 789-803. . 
[33] ISO/IEC guide 99-12:2007, International Vocabulary of Metrology - Basic and General Concepts and Associated Terms, VIM, (International Organization for Standardization, Geneva (Switzerland), 2007).

[34] H. Tada, P.C. Paris and G.R. Irwin, The stress analysis of cracks handbook, Del Research Corporation, Second Edition, 2.2.

[35] A.M.K. Adib and C.A.R.P. Baptista, An exponential equation of fatigue crack growth in titanium, Mat. Sci. Eng. A452-453 (2007) 321-325.

[36] F.A. McClintock, Discussion to C. Laird's paper "The influence of metallurgical microstructure on the mechanisms of fatigue crack propagation," Proceedings Fatigue crack propagation, STP 415, (ASTM, Philadelphia (USA), 1967), 170-174.

[37] D.R.J. Owen and A.J Fawkes, Engineering Fracture Mechanics: Numerical methods and applications, (Pineridge Press, Swansea (UK), 1983).

[38] J. R. Rice, Mechanics of crack tip deformation and extension by fatigue, Proceedings Fatigue crack propagation, STP 415, (ASTM, Philadelphia (USA), 1967), 247-309.

[39] A. Maynadier, M. Poncelet, K. Lavernhe and S. Roux, One-shot measurement of thermal and kinematic fields: Infra-Red Image Correlation (IRIC), Exp. Mech. (2011) doi:10.1007/s11340-011-9483-2.

[40] M. A. Sutton, J. D. Helm and M. L. Boone, Experimental study of crack growth in thin sheet 2024-T3 aluminum under tension-torsion loading, Int. J. Fract. 109 (2001) 285-301. 


\section{List of Tables}

1 Identified coefficients for Paris' law without threshold (a) or with threshold (b). Coefficients for the law without threshold are compared to published values for another titanium grade [35]. Units: $a$ in $\mathrm{mm}, \Delta K$ in $\mathrm{MPa} \sqrt{\mathrm{m}} \ldots \ldots \ldots 23$ 
Table 1: Identified coefficients for Paris' law without threshold (a) or with threshold (b). Coefficients for the law without threshold are compared to published values for another titanium grade [35]. Units: $a$ in $\mathrm{mm}, \Delta K$ in $\mathrm{MPa} \sqrt{\mathrm{m}}$.

\begin{tabular}{|l|c|c|}
\hline Parameter & $A$ & $m$ \\
\hline Post-processing & $4.76 \times 10^{-8}$ & 3.12 \\
I-DIC & $4.65 \times 10^{-8}$ & 3.12 \\
Adib et al. & $7.49 \times 10^{-8}$ & 2.95 \\
\hline
\end{tabular}

(a)

\begin{tabular}{|l|c|c|c|}
\hline Parameter & $B$ & $\Delta K_{t h}$ & $p$ \\
\hline Post-processing & $2.87 \times 10^{-6}$ & 4.12 & 1.77 \\
I-DIC & $1.48 \times 10^{-6}$ & 3.46 & 2.00 \\
\hline
\end{tabular}

(b) 


\section{List of Figures}

1 (a) Image capture during the load history. (b) Dimensions and local detail of the CCT sample. Reference results for these samples $[34,36]$ often use solutions with a centered crack. . . . . . . 27

2 Test configuration for the cyclic loading. A telecentric lens is used to minimize artifacts induced by out-of-plane motions. . . . 28

3 Measured vertical displacement field (in pixels) around the crack tip with Q4-DIC (16-pixel elements are used). No mask was used in this preliminary analysis.

4 (a) Coefficient of order $n=1\left(K_{I}\right)$ measured by both techniques with respect to prescribed value $K_{I}$ for the a priori analysis. (b) Error between measured and prescribed $K_{I}$ values for both techniques. The measurement error for I-DIC remains small up to values of the order of $0.05 \mathrm{MPa} \sqrt{\mathrm{m}}$ whereas it is more important up to values of $1 \mathrm{MPa} \sqrt{\mathrm{m}}$ for the post-processing route. . . . . . 3

5 Offset between identified and prescribed crack tip positions for both techniques (in pixels). Crack tip positions cannot be trusted for $K_{I}$ levels less than $0.05 \mathrm{MPa} \sqrt{\mathrm{m}}$ for I-DIC with the selected mask parameters (inner radius: 10 pixels, external radius: 400 pixels). It is clearly more delicate for the post-processing route. . 31 
6 Measured displacement field with Q4-DIC (center), extracted field with the post-processing technique (top), measured field with I-DIC (bottom) and residuals (right). For the post-processing technique, residuals are the raw differences between the measured displacement field with Q4-DIC and the extracted field (for the two directions). For integrated-DIC, residuals are gray level differences $|f(\mathbf{x})-g(\mathbf{x}+\mathbf{u}(\mathbf{x}))|$ normalized by the dynamic range of the reference picture. . . . . . . . . . . . . . . . . . 32

7 (a) Gray level residuals $|f(\mathbf{x})-g(\mathbf{x}+\mathbf{u}(\mathbf{x}))|$ as percentage of the dynamic range of the reference picture for integrated-DIC (left) and the post-processing technique (right). The displacement field $\mathbf{u}$ is for each technique obtained by multiplying the reference fields (6) and (7) by the measured amplitudes $\omega_{n}$ and $v_{n}$. (b) Mean gray level residuals normalized by the dynamic range of the reference picture for both techniques. The increase of the measured amplitudes makes the correlation residuals increase for the post-processing technique but not for I-DIC. . . . . . . . . . 33

8 (a) Measured $\Delta a$ (in $\mathrm{mm}$ ) as a function of the number of cycles for both methods. (b) Measured $\Delta K$ as a function of the crack length increment $\Delta a$ for both methods compared to tabulated values for CCT samples [34], and interpolated curves obeying Equation (13). . . . . . . . . . . . . . . . . . . . 34

9 Measured and fitted crack growth increment without and with a threshold in Paris' law for I-DIC (a) and post-processing (b)

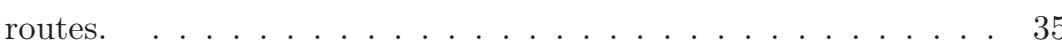


10 Raw measurement (symbols) and interpolation (solid lines) for Paris' law without threshold (a) and with threshold (b). Results without threshold are compared to reported values of another titanium grade $[35] . \ldots \ldots \ldots \ldots$. . . . . . . . . . 36

$11 T$-stress estimation as a function of the number of cycles for both techniques, and for tabulated values $[36] \ldots \ldots \ldots \ldots$

12 Estimates of the size and shape of the plastic zone. (a) Plastic zone using only $K_{I}, K_{I}$ and $T$-stress components or all the contributions for the last analyzed picture. (b) Estimation of the plastic zone radius. I-DIC provides a gradually increasing value whereas the post-processing approach seems not to be able to measure meaningful $\omega_{-3}$ amplitudes for the first 40 pictures. Note that the plastic zone estimates provided by DIC are adjusted over the first 40 pictures (i.e. 40,000 cycles), since they are defined up to a multiplicative constant [Equation (17)] . . . . . . . . . . 38

13 Displacement amplitude (in pixels) as a function of the distance from the crack tip along the ligament, for image 40. The gray zones correspond to masked areas that are not taken into account in the analysis. The black solid line is the sum of all plotted contributions. 


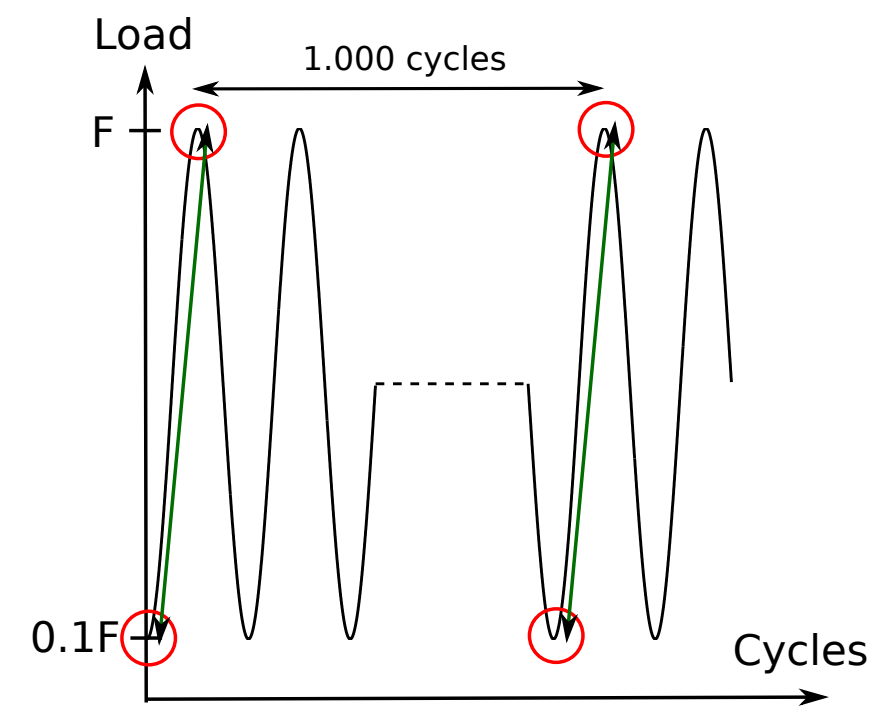

(a)

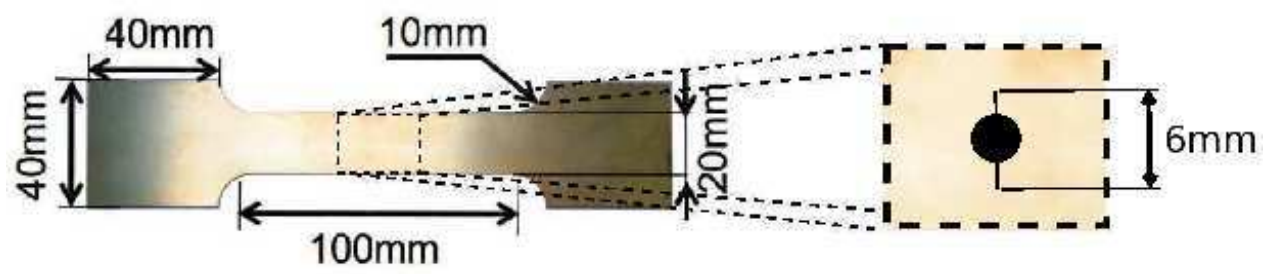

(b)

Figure 1: (a) Image capture during the load history. (b) Dimensions and local detail of the CCT sample. Reference results for these samples [34,36] often use solutions with a centered crack. 


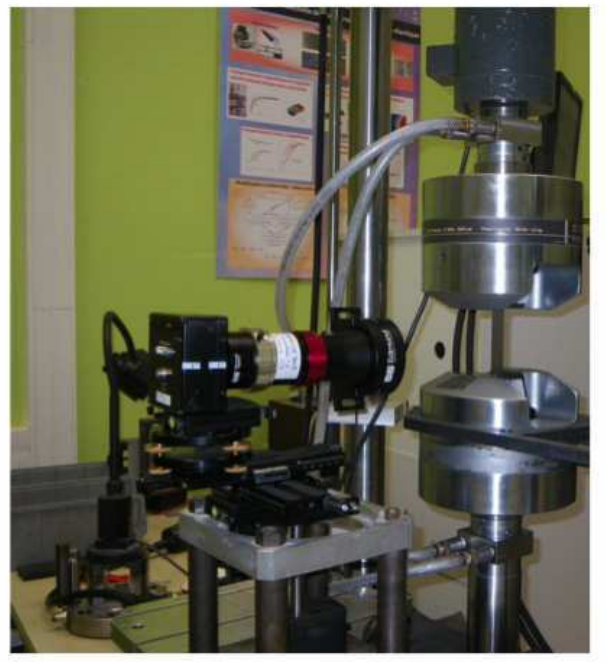

Figure 2: Test configuration for the cyclic loading. A telecentric lens is used to minimize artifacts induced by out-of-plane motions. 


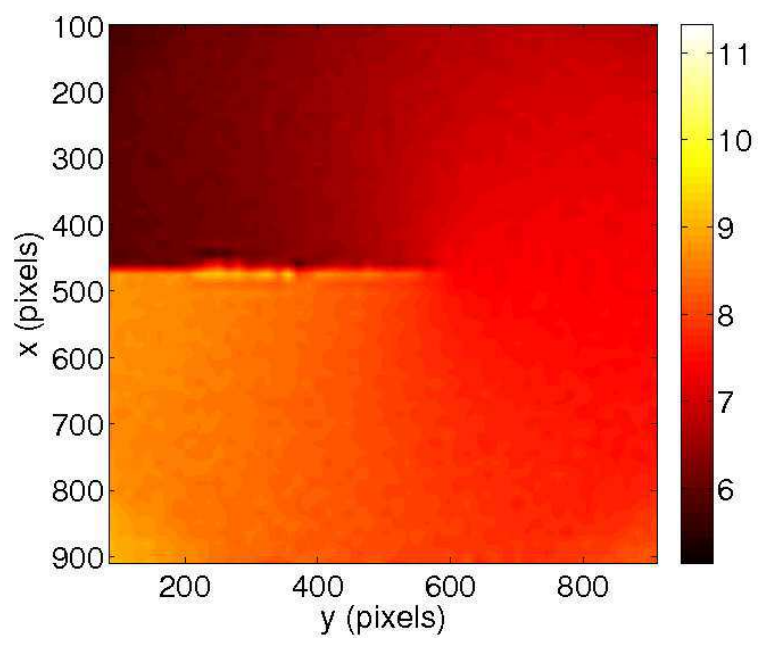

Figure 3: Measured vertical displacement field (in pixels) around the crack tip with Q4-DIC (16-pixel elements are used). No mask was used in this preliminary analysis. 


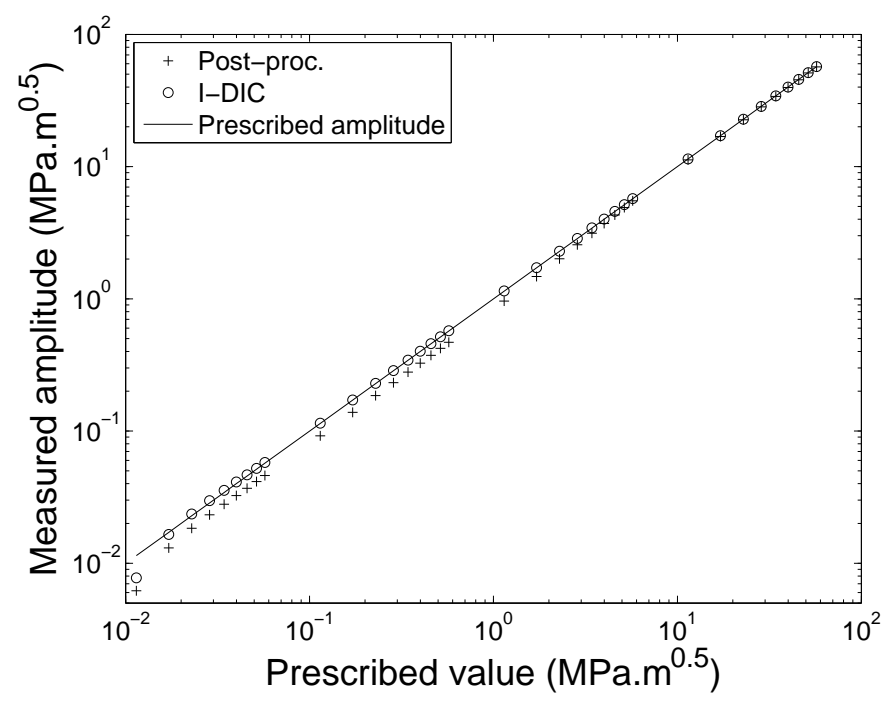

(a)

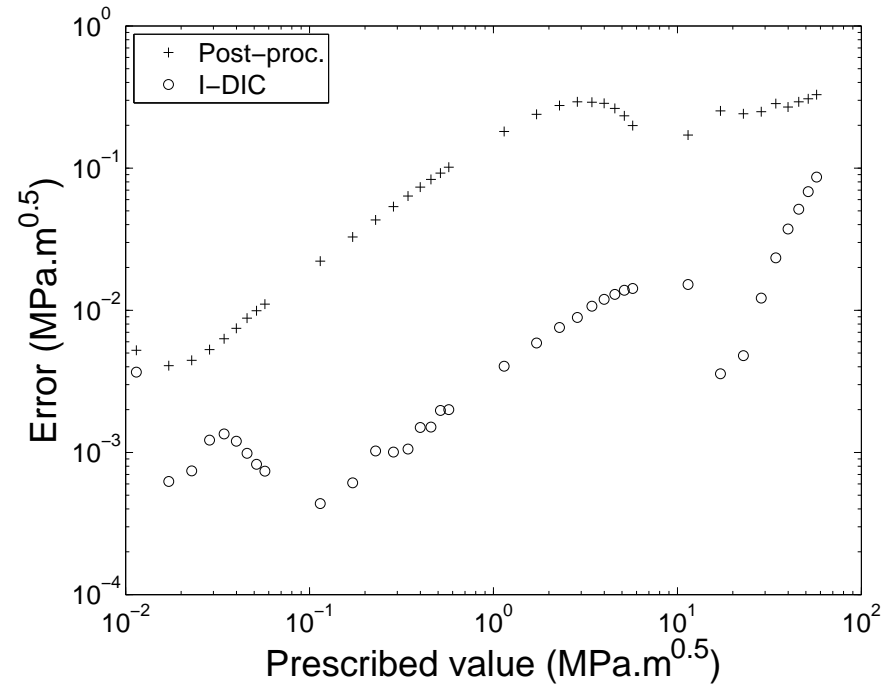

(b)

Figure 4: (a) Coefficient of order $n=1\left(K_{I}\right)$ measured by both techniques with respect to prescribed value $K_{I}$ for the a priori analysis. (b) Error between measured and prescribed $K_{I}$ values for both techniques. The measurement error for I-DIC remains small up to values of the order of $0.05 \mathrm{MPa} \sqrt{\mathrm{m}}$ whereas it is more important up to values of $1 \mathrm{MPa} \sqrt{\mathrm{m}}$ for the post-processing route. 


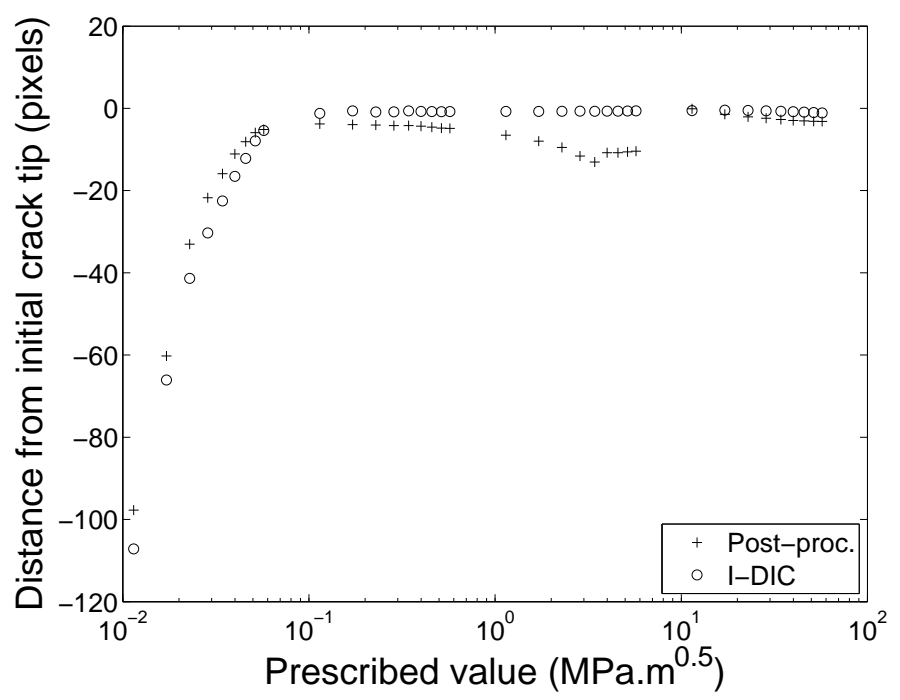

Figure 5: Offset between identified and prescribed crack tip positions for both techniques (in pixels). Crack tip positions cannot be trusted for $K_{I}$ levels less than $0.05 \mathrm{MPa} \sqrt{\mathrm{m}}$ for I-DIC with the selected mask parameters (inner radius: 10 pixels, external radius: 400 pixels). It is clearly more delicate for the post-processing route. 


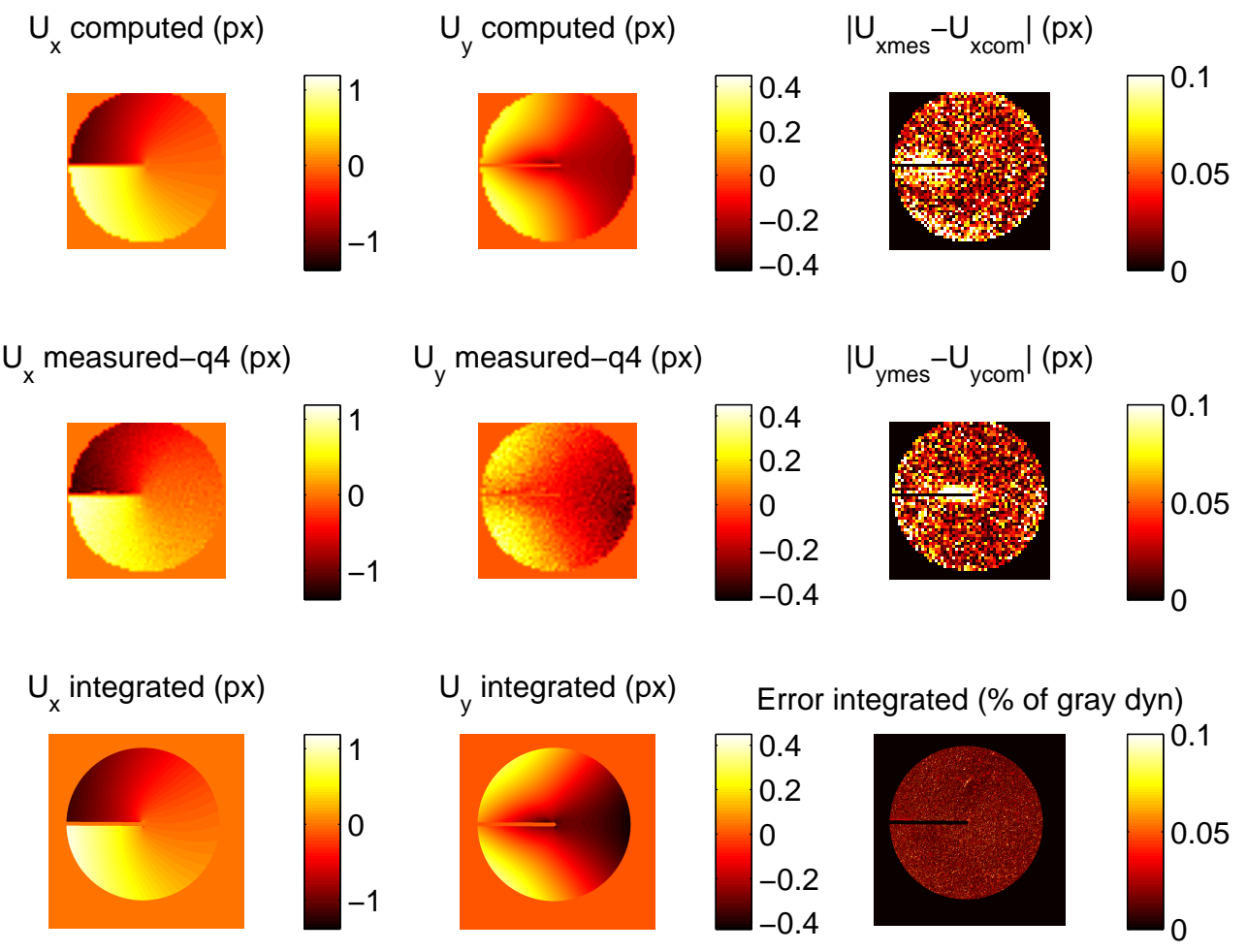

Figure 6: Measured displacement field with Q4-DIC (center), extracted field with the postprocessing technique (top), measured field with I-DIC (bottom) and residuals (right). For the post-processing technique, residuals are the raw differences between the measured displacement field with Q4-DIC and the extracted field (for the two directions). For integrated-DIC, residuals are gray level differences $|f(\mathbf{x})-g(\mathbf{x}+\mathbf{u}(\mathbf{x}))|$ normalized by the dynamic range of the reference picture. 

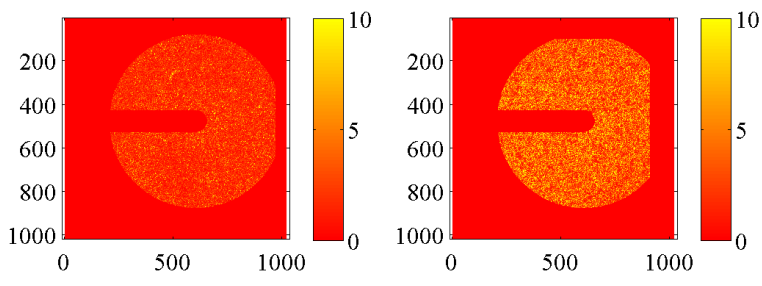

(a)

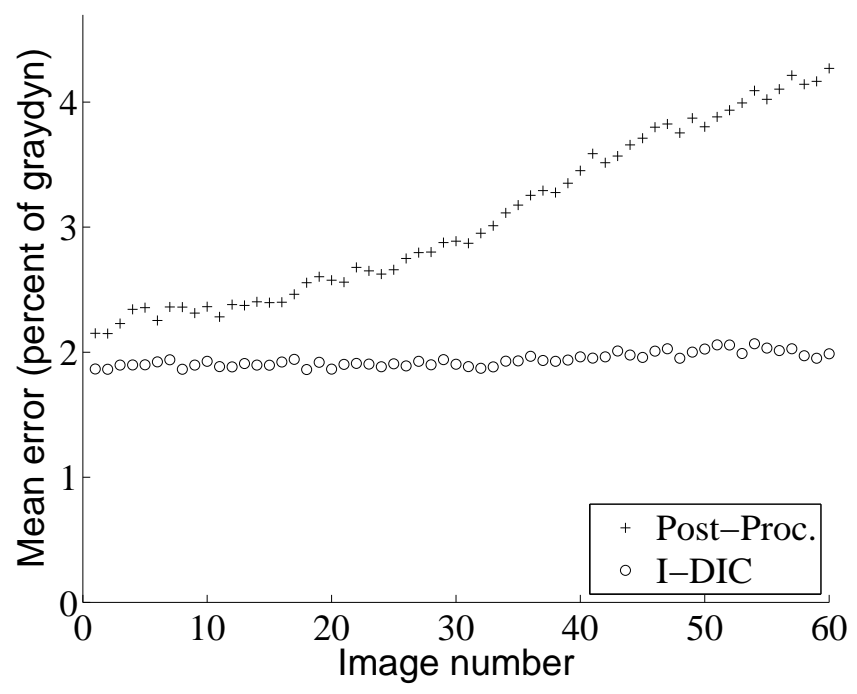

(b)

Figure 7: (a) Gray level residuals $|f(\mathbf{x})-g(\mathbf{x}+\mathbf{u}(\mathbf{x}))|$ as percentage of the dynamic range of the reference picture for integrated-DIC (left) and the post-processing technique (right). The displacement field $\mathbf{u}$ is for each technique obtained by multiplying the reference fields (6) and (7) by the measured amplitudes $\omega_{n}$ and $v_{n}$. (b) Mean gray level residuals normalized by the dynamic range of the reference picture for both techniques. The increase of the measured amplitudes makes the correlation residuals increase for the post-processing technique but not for I-DIC. 


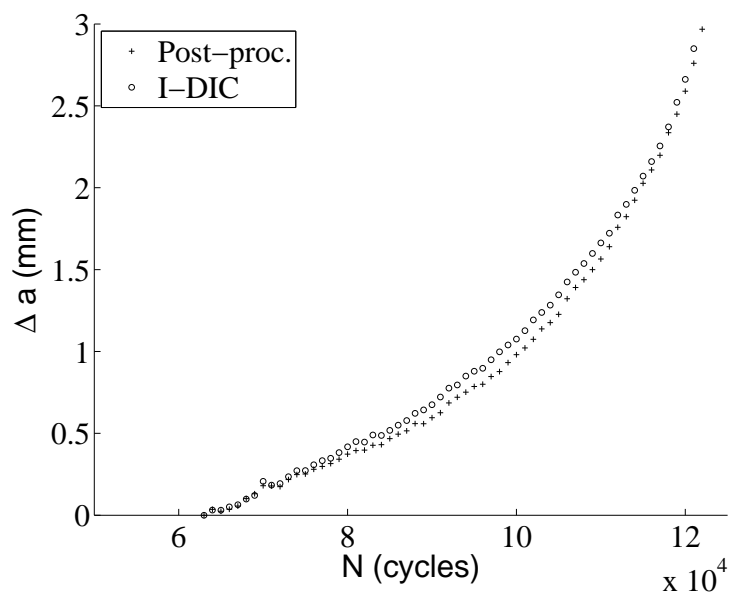

(a)

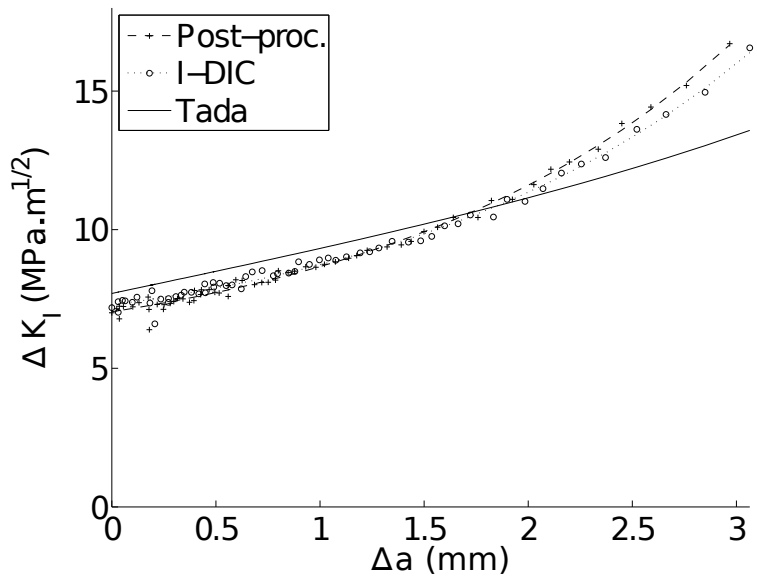

(b)

Figure 8: (a) Measured $\Delta a$ (in $\mathrm{mm}$ ) as a function of the number of cycles for both methods. (b) Measured $\Delta K$ as a function of the crack length increment $\Delta a$ for both methods compared to tabulated values for CCT samples [34], and interpolated curves obeying Equation (13). 


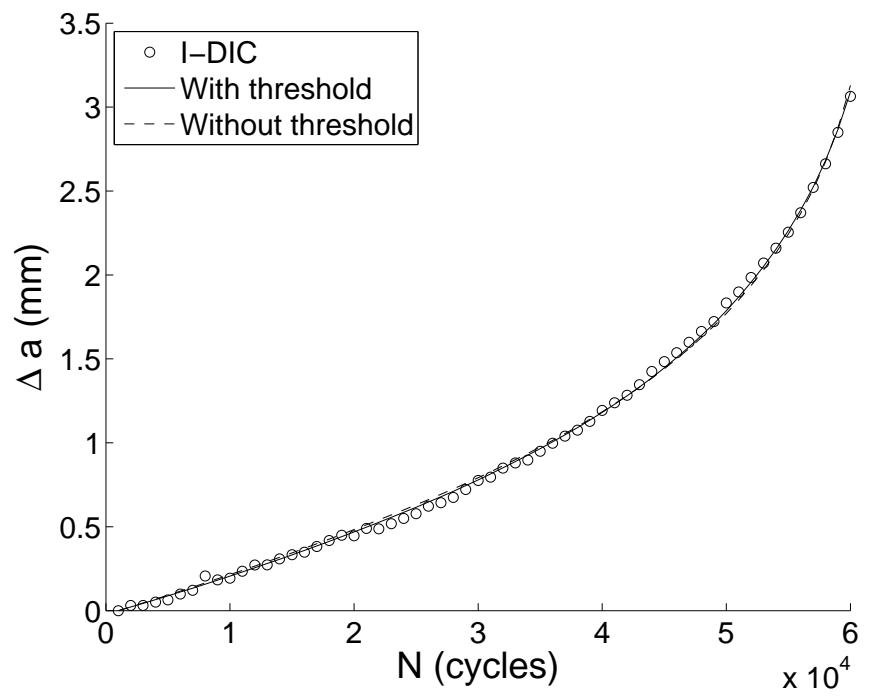

(a)

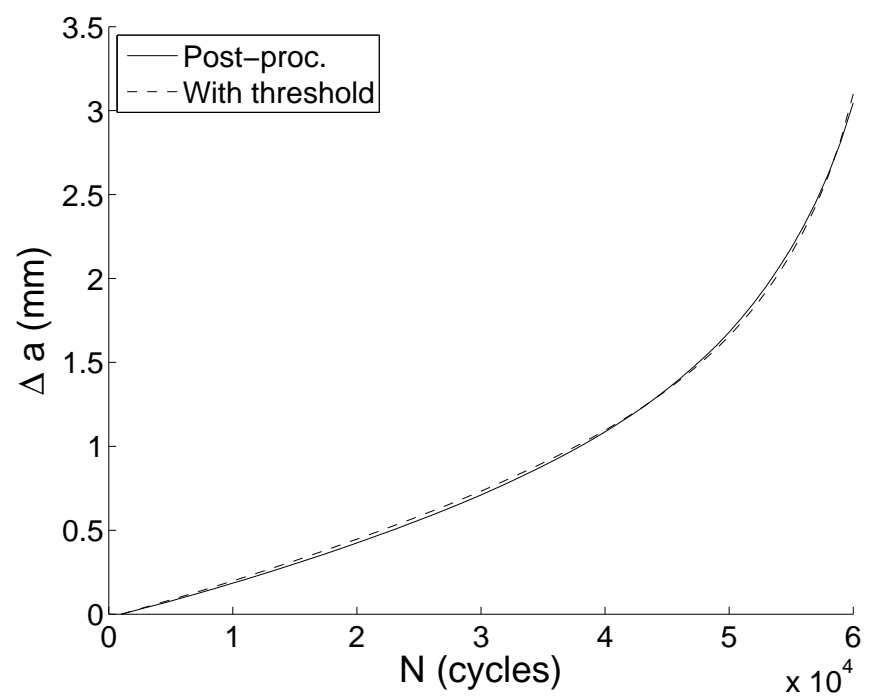

(b)

Figure 9: Measured and fitted crack growth increment without and with a threshold in Paris' law for I-DIC (a) and post-processing (b) routes. 


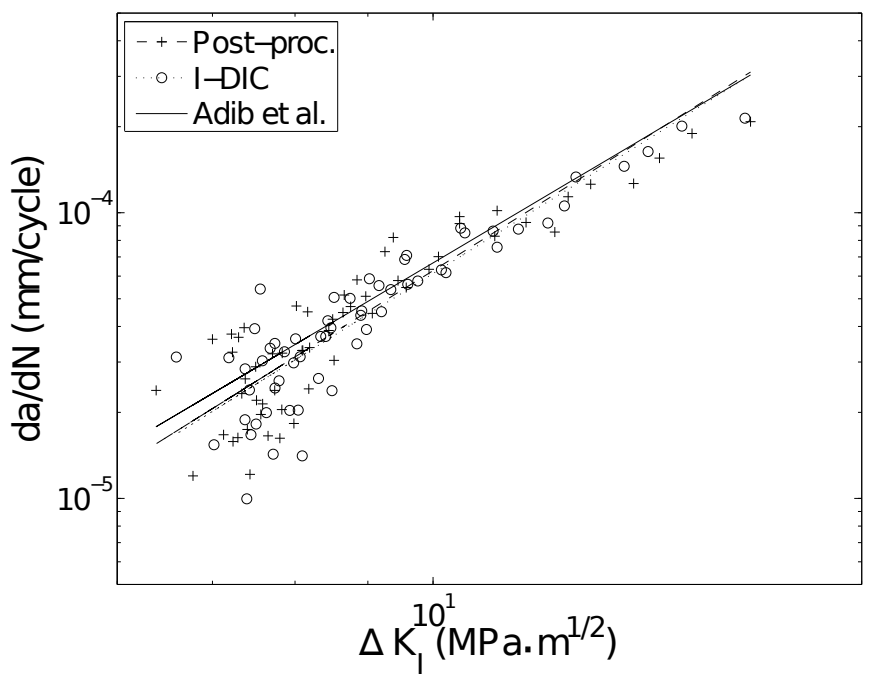

(a)

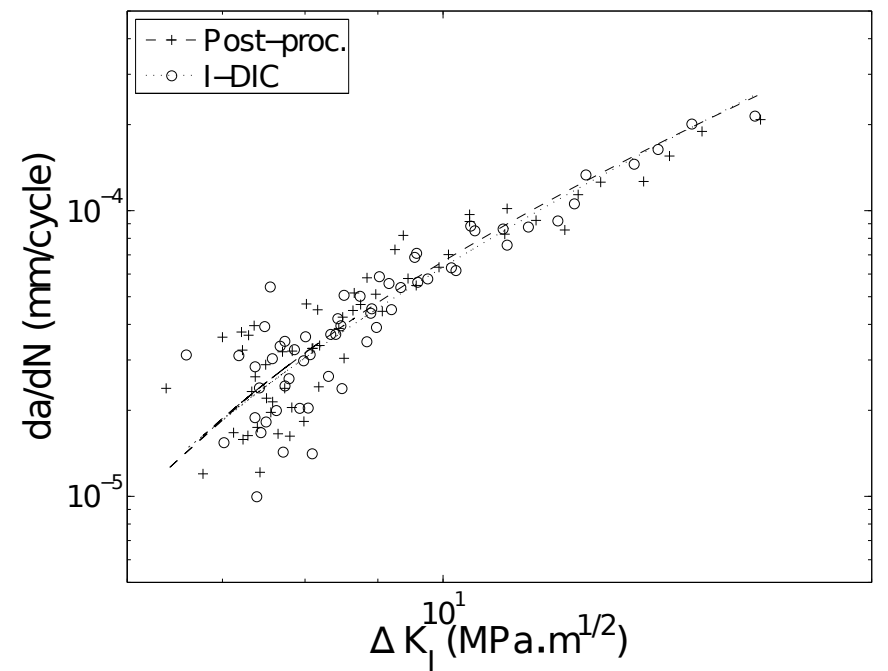

(b)

Figure 10: Raw measurement (symbols) and interpolation (solid lines) for Paris' law without threshold (a) and with threshold (b). Results without threshold are compared to reported values of another titanium grade [35]. 


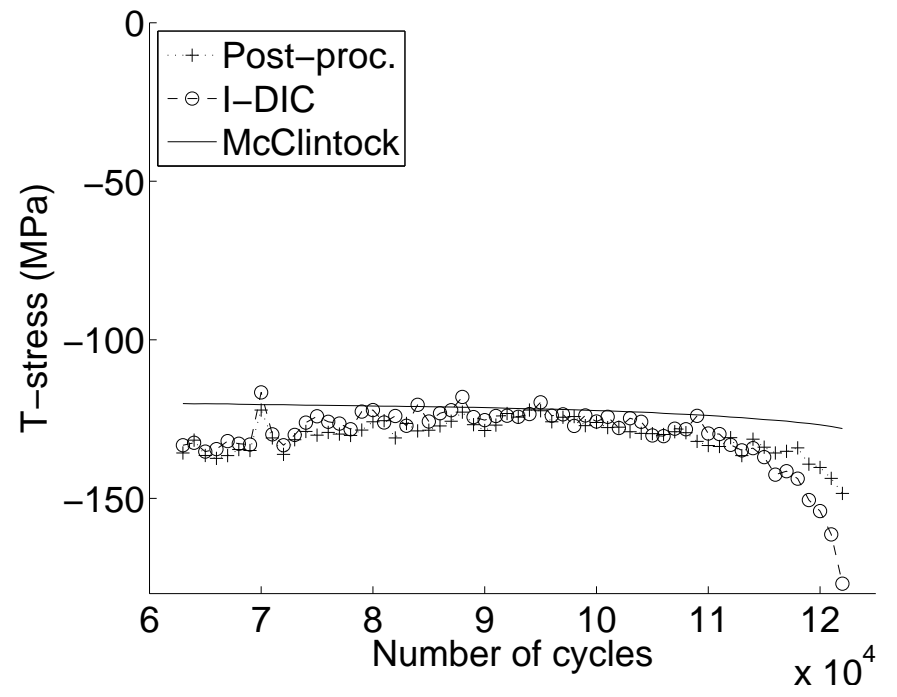

Figure 11: $T$-stress estimation as a function of the number of cycles for both techniques, and for tabulated values [36]. 


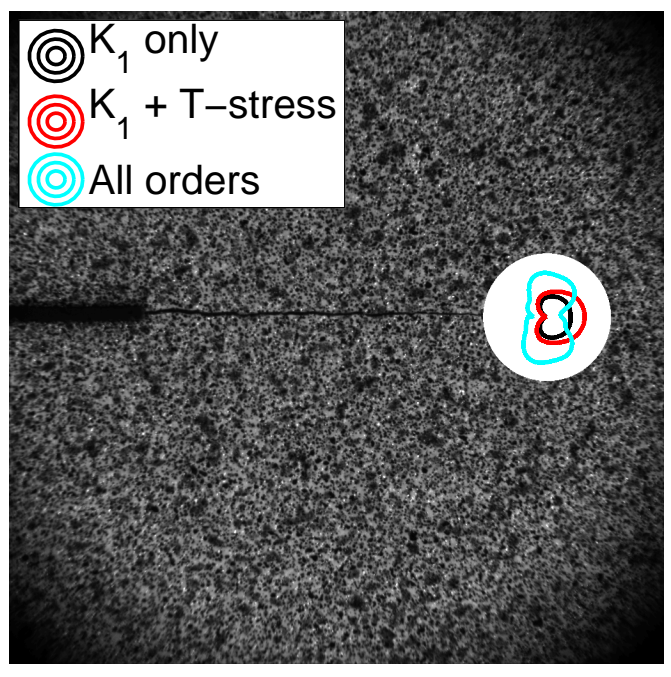

(a)

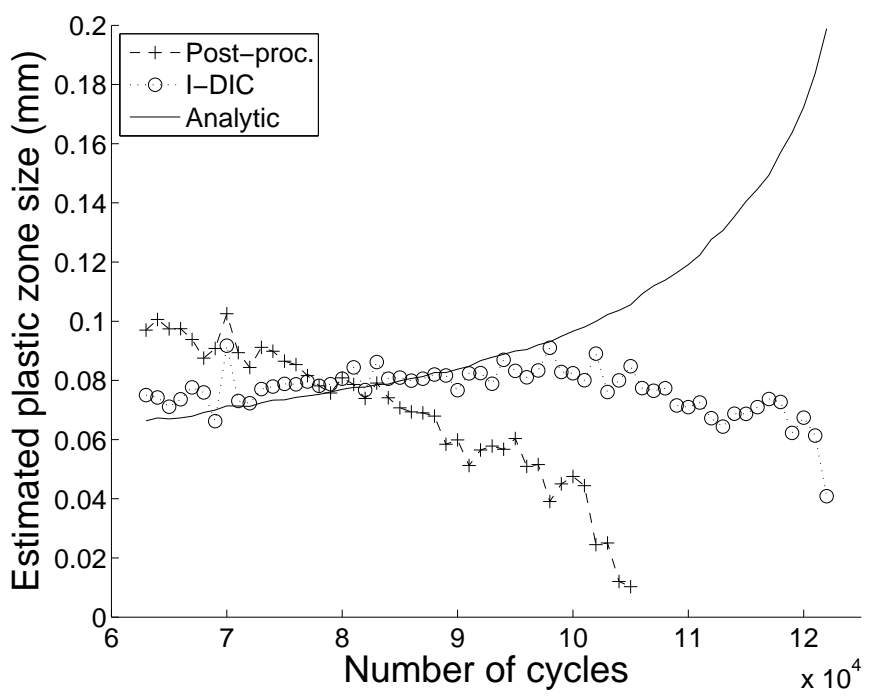

(b)

Figure 12: Estimates of the size and shape of the plastic zone. (a) Plastic zone using only $K_{I}, K_{I}$ and $T$-stress components or all the contributions for the last analyzed picture. (b) Estimation of the plastic zone radius. I-DIC provides a gradually increasing value whereas the post-processing approach seems not to be able to measure meaningful $\omega_{-3}$ amplitudes for the first 40 pictures. Note that the plastic zone estimates provided by DIC are adjusted over the first 40 pictures (i.e. 40,000 cycles), since they are defined up to a multiplicative constant [Equation (17)]. 


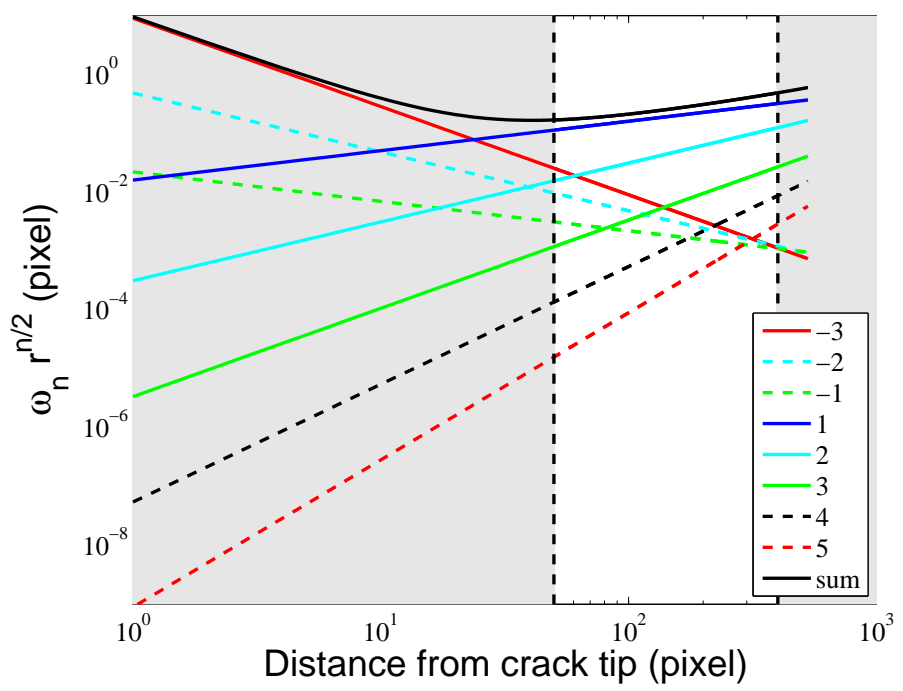

Figure 13: Displacement amplitude (in pixels) as a function of the distance from the crack tip along the ligament, for image 40. The gray zones correspond to masked areas that are not taken into account in the analysis. The black solid line is the sum of all plotted contributions. 